\title{
STOCHASTIC ANALYSIS TO ASSESS THE PERFORMANCE OF PELTON WHEEL TURBINE KEEPING HEAD CONSTANT
}

\author{
Daniel Meneses $^{1}$, SampadaPednekar $^{2}$, AmeyPatil $^{3}$, NeenaPanandikar ${ }^{4}$ \\ ${ }^{1,2,3,4}$ Department of Mechanical Engineering, Padre Conceicao College of Engineering, Verna Goa \\ Tel no: +919049160677, email: daniel.meneses160@gmail.com
}

\begin{abstract}
The study uses stochastic approach to assess the performance of a Pelton wheel turbine. The performance of Pelton Wheel turbine is affected by various inputs which when optimized produce a desirable output. Stochastic studies take into account the variations that are not visualized in the practical experiment. The use of Response surface curves further helps evaluate and optimize the set of inputs to produce maximum efficiency. The relations developed by the equations obtained from ANOVA techniques and the theoretical equations show close similarities suggesting a small percent of error in the Pelton wheel test rig which is assessed accordingly.
\end{abstract}

Keywords: Stochastic, Monte Carlo Simulation, Response Surface Methodology

\section{INTRODUCTION}

Pelton Wheel turbine is a type of hydraulic impulse turbine. It is a type of tangential flow turbine wherein water flows tangent to the wheel blades. The blades used are double half shaped buckets stuck adjacent to each other. Many of such buckets are equally spaced around the wheel. The power developed is purely from change in kinetic energy, the pressure remaining atmospheric throughout. Water from the dam flows down through the penstock. Sometimes there is an intermediate reservoir between the dam and the turbine to store water in times of scarcity. The water then flows through the jet nozzles to the buckets impacting them which in turn rotate the turbine which is coupled to a generator to produce electricity.

The use of stochastic analysis is to randomly generate input parameters. These factors when combined randomly produce efficiencies usually in the optimal range. Stochastic analysis considers the factors affecting performance to follow a probability distribution rather than a deterministic value. Monte Carlo Simulationis an associated technique which aids in the stochastic process. Monte Carlo process can be conducted in Excel using the random number generator function.

There are a number of factors that influence the performance of a Pelton turbine ranging from number of buckets to turbine speed. The Pelton test rig used in our college has a set of four input factors namely: head, pressure, turbine speed and turbine load. These set of inputs decide the performance of the Pelton test rig. The variation of these inputs yields a specific set of responses which are analyzed further using response curves. The responses/outputs considered are: head in meters of water, pump pressure, discharge, brake power and most importantly the test rig efficiency. For the experiment, the turbine head is kept constant while the others factors are varied according to the trend they follow. It is found that as head is kept constant, increasing load decreases the speed on turbine.

Response surface methodology is an optimization technique. It makes use of ANOVA tools to generate the equations in terms of input parameters. The experiment is modeled according to the requirements and the output functions are further developed. The relation may be of any order depending on the suggested $\mathrm{R}$ value. Response curves show the variation of the parameters for a specific output. Response surface is a type of interpolation optimization technique which interpolates the response for optimal set of inputs. The optimal set is further verified on the Pelton wheel test rig to assess its performance.

The responses obtained from ANOVA equations and the theoretical equations are tested for the optimal set which gives the error in the test rig. Errors in a Pelton wheel have various sources. The main sources of error are vibrations of the setup. Frictional loses are also significant which affect the performance of the test rig. The losses prove significant when assessed against optimal set. The test rig needs its occasional maintenance to keep the errors as low as possible. Error is likely to creep in the test rig as a result of poor handling and care which ultimately affect its performance and efficiency. The ultimate goal of the experiment is to set the margin between the errors and suggest measures.

Stochastic studies have a wide scope and be used for a number of system running under different operating conditions, stochastic process is merely a technique to avoid variations and unaccounted losses and assess the system. The scope of stochastic analysis ranges wide to infinite bounds and finds its use in every industry from risk management in economics to performance analysis is engineering systems. 


\section{METHODOLOGY}

\subsection{The Monte Carlo Simulation}

Monte Carlo Simulation is probabilistic type of experiment that uses random sampling to obtain numerical results. Their essential idea is using randomness to solve problems that might be deterministic in principle. For the purpose of the project we are performing Monte Carlo process in Microsoft Excel. A set of 1000 runs are used for which the inputs follow a random trend. The random number generator function is used given by- RAND ()*difference+ initial value

The outputs are determined according to existing relations. This randomized experiment provides us the various set of inputs producing outputs in infinite range.

The input ranges are given below:

1. Head- 7 to $14 \mathrm{~cm}$

2. Pressure- 2 to $7 \mathrm{~kg} / \mathrm{cm}^{\wedge} 2$

3. Load- 0.1 to $\mathrm{kgf}$

4. Speed- 600 to $1200 \mathrm{rpm}$

The efficiencies are found to cross the limit of $100 \%$ as there is no limit set on outputs. As Monte Carlo simulation is randomized technique, the outputs are bound to cross their natural limits.

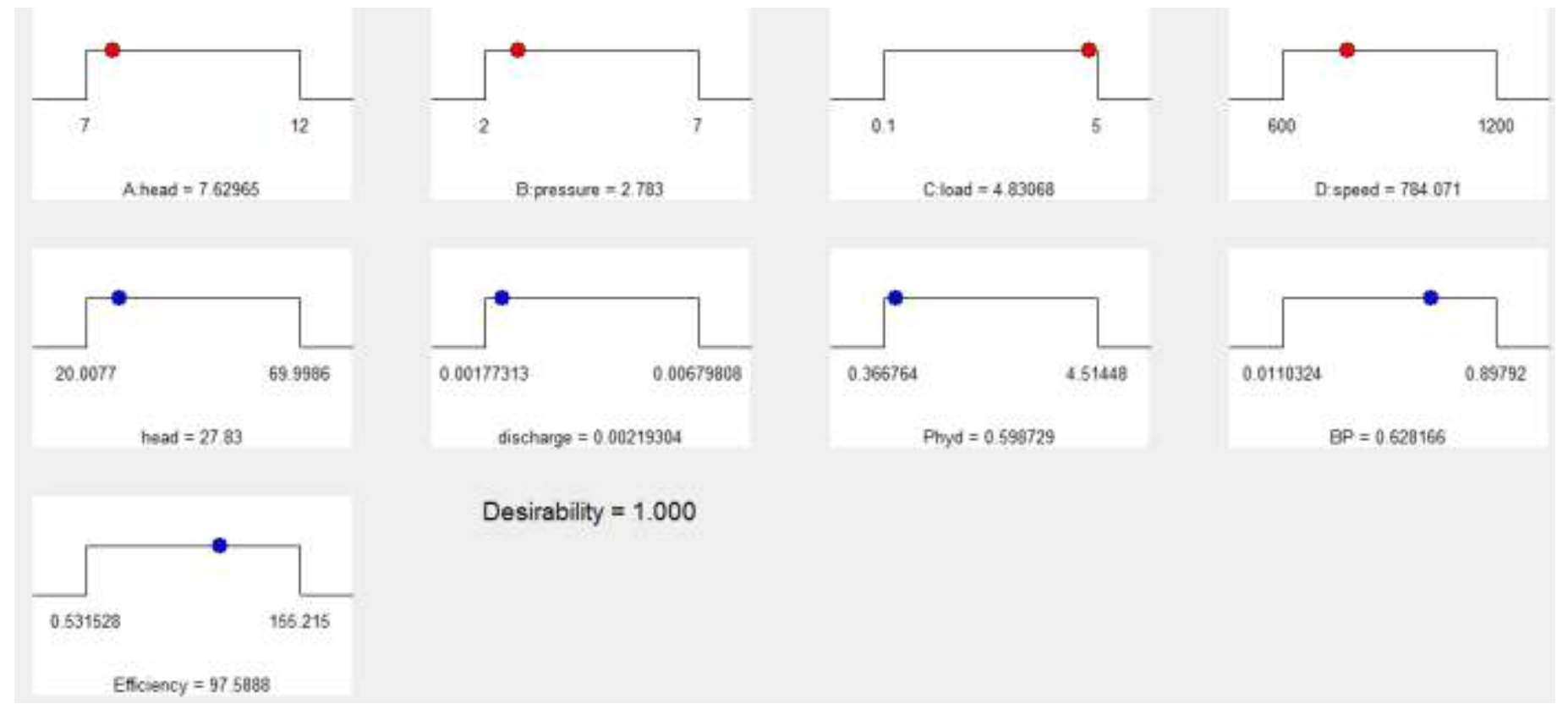
Fig 2. Optimal set obtained from Design expert using Monte Carlo technique

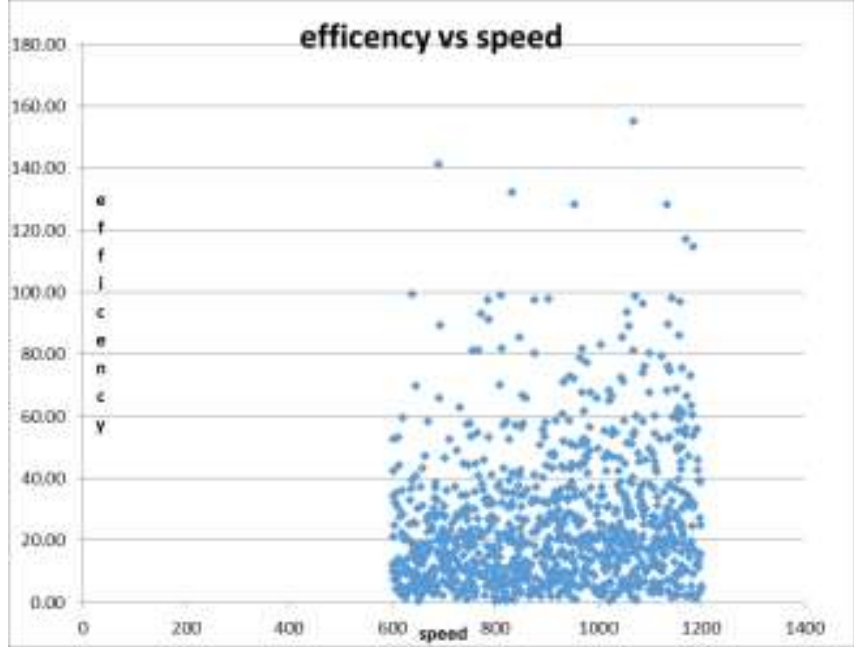

Fig1. Monte Carlo Simulation for a set of 1000 runs in Microsoft Excel.

\subsection{Response Curves For Stochastic Process}

The abovesets of readings are fed into optimization software to develop the response curves. The optimal set is then studies for this stochastic experiment. The ANOVA relations are seen to follow fifth or sixth order models which give complex equations. The optimal sets of inputs are then viewed which give the highest efficiencies. The response surface curves are then developed for a particular output and a resulting relation is drawn. Response surface methodology of a stochastic process tends to interpolate responses further to infinite bounds; hence the outputs must be kept in range to limit the maximum output range. The response of interest is primarily the efficiency which cannot cross a value of 100 practically; therefore a check needs to be maintained for the efficiency. The response curves for the various outputs are depicted below: 


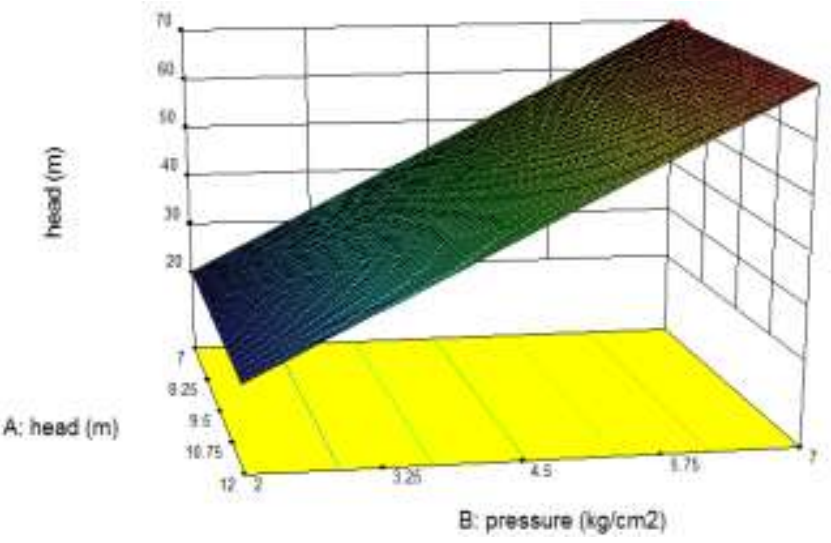

Fig3.3D surface curve for head

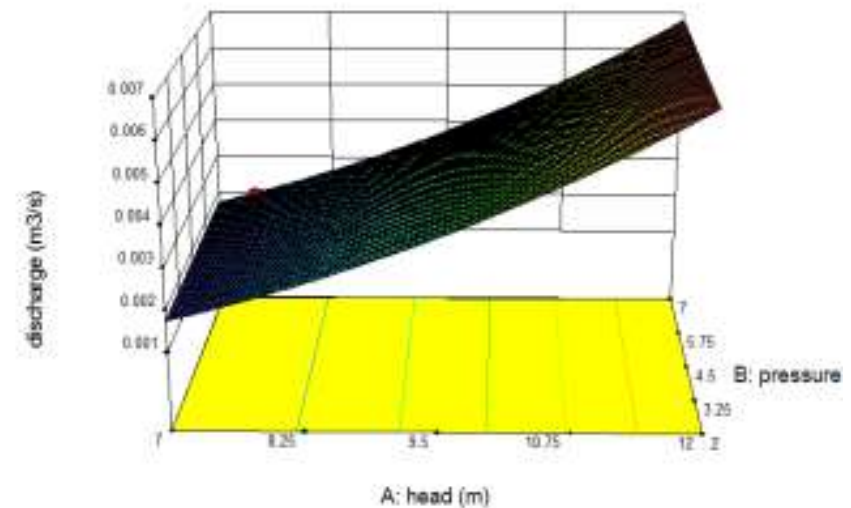

Fig4. 3D surface curve for discharge

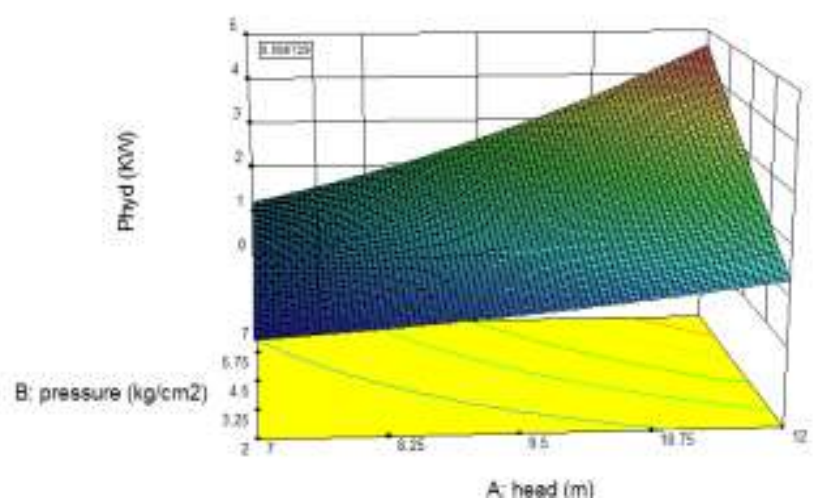

Fig5. 3D surface curve for hydraulic power

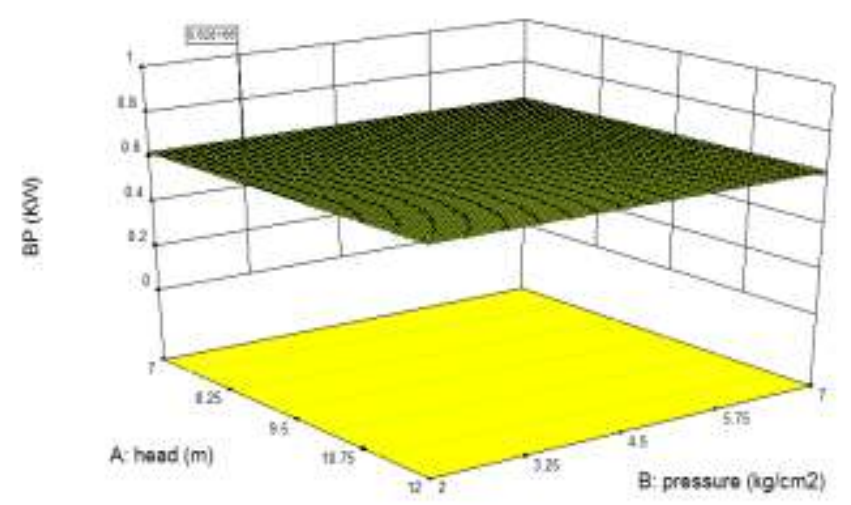

Fig6. 3D surface curve for brake power

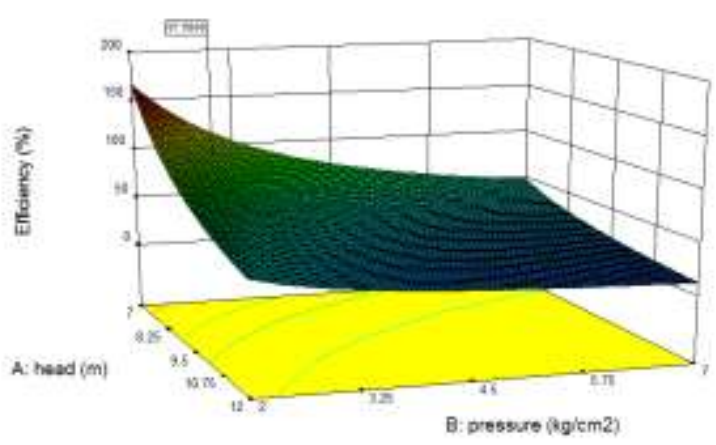

Fig7. 3D surface curve for efficiency

The optimal set suggested a likely efficiency of $97.58 \%$ efficiency which is plotted on the graphs above.

\subsection{The Practical Experiment}

The second phase of our experiment deals with practically varying the inputs on test rig and thereby calculating the efficiency. This second part is entirely based on the readings obtained from the Pelton wheel test rig. Existing empirical formulas are used to calculate the final efficiency. The Pelton test rig is operated for five times in a week and the readings are noted down. Keeping a time lag between subset of readings helps minimize the possibility of errors due to environmental conditions. The Pelton wheel turbine test rig specifications are given below:

- $\quad$ Supply pump- 7.5hp, 3ph, 440v, 50Hz, AC

- Turbine
a) Mean diameter- $259 \mathrm{~mm}$
b) Number of buckets- 20
c) Diameter of jet- $18 \mathrm{~mm}$
d) Head- $100 \mathrm{~m}$

- $\quad$ Loading- brake drum

- Maximum shaft output- $1.5 \mathrm{KW}$, Vnotch=60deg, $\mathrm{Cd}=0.6$

The experiment is performed keeping head constant throughout the subset of readings. A set of 21 readings are taken for varying levels of speed, pressureand load andthe outputs are calculated form empirical formulas. The readings taken are tabulated in Microsoft Excel and shown below: 


\begin{tabular}{|c|c|c|c|c|c|c|c|c|}
\hline \multicolumn{9}{|c|}{ Inputs for constant head } \\
\hline \multicolumn{3}{|c|}{ head h Pressure $\mathrm{speed}$} & \multirow{2}{*}{\begin{tabular}{|l|} 
Ioad \\
0.4 \\
\end{tabular}} & $\mathrm{H}$ & Discharge $\mathrm{m}^{\wedge} 3 / \mathrm{s}$ & Phyd KW & BP KW & $n$ \\
\hline 0.11 & 2 & 1000 & & 20 & 0.0033 & 0.642844 & 0.061607 & 9.583472 \\
\hline 0.11 & 2 & 900 & 0.95 & 20 & 0.0033 & 0.642844 & 0.131685 & 20.48467 \\
\hline 0.11 & 2 & 850 & 1.45 & 20 & 0.0033 & 0.642844 & 0.189826 & 29.52907 \\
\hline 0.11 & 2 & 750 & 1.9 & 20 & 0.0033 & 0.642844 & 0.219474 & 34.14112 \\
\hline 0.11 & 2 & 600 & 2.6 & 20 & 0.0033 & 0.642844 & 0.240267 & 37.37554 \\
\hline 0.12 & 3 & 1355 & 0.3 & 30 & 0.0040 & 1.188838 & 0.062608 & 5.266313 \\
\hline 0.12 & 3 & 1285 & 0.9 & 30 & 0.0040 & 1.188838 & 0.178121 & 14.98276 \\
\hline 0.12 & 3 & 1099 & 1.8 & 30 & 0.0040 & 1.188838 & 0.304676 & 25.62809 \\
\hline 0.12 & 3 & 969 & 2.9 & 30 & 0.0040 & 1.188838 & 0.432803 & 36.40557 \\
\hline 0.12 & 3 & 803 & 4.25 & 30 & 0.0040 & 1.188838 & 0.525622 & 44.21306 \\
\hline 0.133 & 4 & 1550 & 0.2 & 40 & 0.0052 & 2.052018 & 0.047745 & 2.326747 \\
\hline 0.133 & 4 & 1500 & 1.1 & 40 & 0.0052 & 2.052018 & 0.254128 & 12.3843 \\
\hline 0.133 & 4 & 1350 & 1.8 & 40 & 0.0052 & 2.052018 & 0.374261 & 18.23869 \\
\hline 0.133 & 4 & 1080 & 4 & 40 & 0.0052 & 2.052018 & 0.665353 & 32.42434 \\
\hline 0.133 & 4 & 1000 & 4.75 & 40 & 0.0052 & 2.052018 & 0.731581 & 35.65176 \\
\hline 0.133 & 4 & 900 & 6 & 40 & 0.0052 & 2.052018 & 0.831692 & 40.53043 \\
\hline 0.137 & 5 & 1735 & 0.2 & 50 & 0.0056 & 2.768078 & 0.053444 & 1.930722 \\
\hline 0.137 & 5 & 1660 & 0.8 & 50 & 0.0056 & 2.768078 & 0.204535 & 7.389047 \\
\hline 0.137 & 5 & 1500 & 1.6 & 50 & 0.0056 & 2.768078 & 0.369641 & 13.3537 \\
\hline 0.137 & 5 & 1520 & 2 & 50 & 0.0056 & 2.768078 & 0.468212 & 16.91469 \\
\hline 0.137 & 5 & 1410 & 2.4 & 50 & 0.0056 & 2.768078 & 0.521194 & 18.82872 \\
\hline
\end{tabular}

Fig8.Pelton test rig readings with constant speed in Microsoft Excel.

A maximum efficiency of $44.21 \%$ was gained from Pelton test rig readings.

\subsection{Response Surface Method}

The readings obtained above are fed into Design Expert; anoptimization response surface software. Design Expert provides an interface to input values from the test rig and consequently develop ANOVA equations which define the optimal set of inputs that define best efficiency.For the purpose of the project we are making use of Design Expert version 10. The ranges for the inputs are defined by the upper and lower bounds from the readings taken from test rig. The models suggested by the software are fairly linear and display a simple linear relation in terms of inputs. The ANOVA equations in terms of main factors are given below:

Final Equation in Terms of Actual Factors:

$$
\begin{gathered}
\text { head }= \\
-8.42757 \mathrm{E}-014 \\
+1.16922 \mathrm{E}-012{ }^{*} \text { head } \\
+10.00000{ }^{*} \text { pressure } \\
-1.63570 \mathrm{E}-017{ }^{*} \text { speed } \\
-3.51768 \mathrm{E}-015{ }^{*} \text { load }
\end{gathered}
$$

Fig9. ANOVA equations for head over notch from Deign Expert
Final Equation in Terms of Actual Factors:

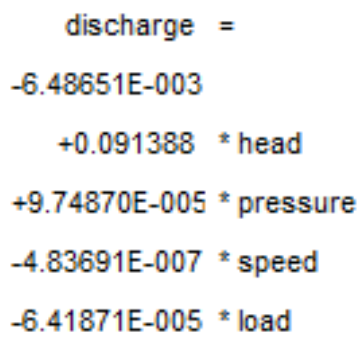

Fig10. ANOVA equations for discharge from Deign Expert

Final Equation in Terms of Actual Factors:

Phyd =

$$
\begin{gathered}
-2.60856 \\
+24.95774 \text { * head } \\
+0.73024{ }^{*} \text { pressure } \\
-9.68167 \mathrm{E}-004{ }^{*} \text { speed } \\
-0.12848 * \text { load }
\end{gathered}
$$

Fig11. ANOVA equations for hydraulic power from Deign Expert 
Final Equation in Terms of Actual Factors:

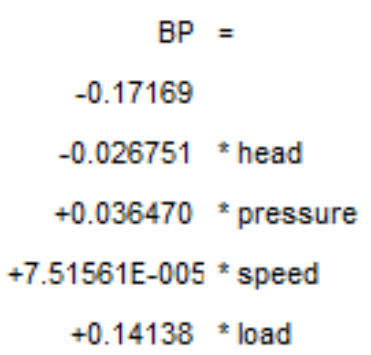

Fig12. ANOVA equations for brake power from Deign Expert

\section{Final Equation in Terms of Actual Factors:}

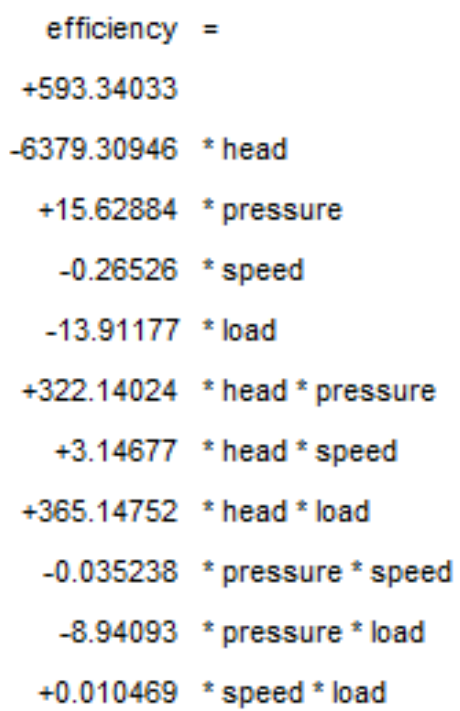

Fig13. ANOVA equations for efficiency from Deign Expert

The response curves for the developed ANIOVA set are depicted below:

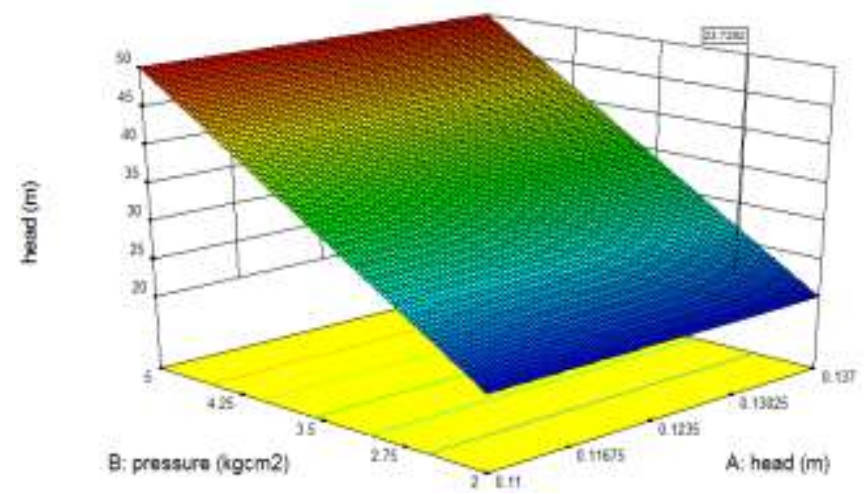

Fig14. Response curve for head

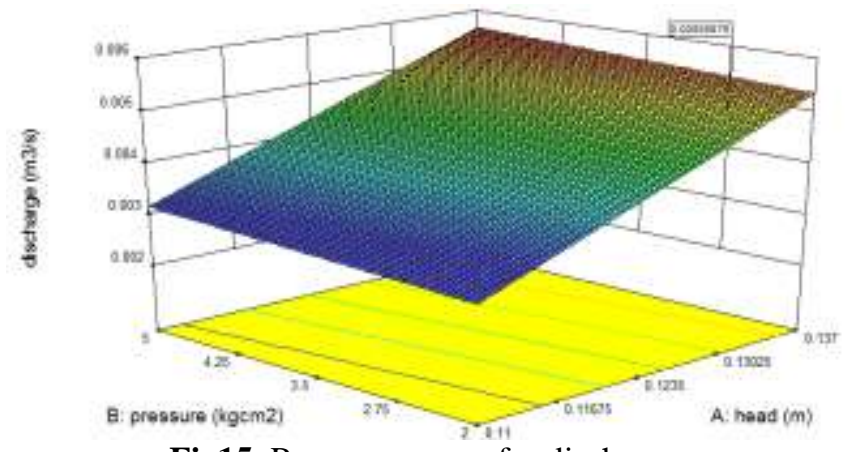

Fig15. Response curve for discharge

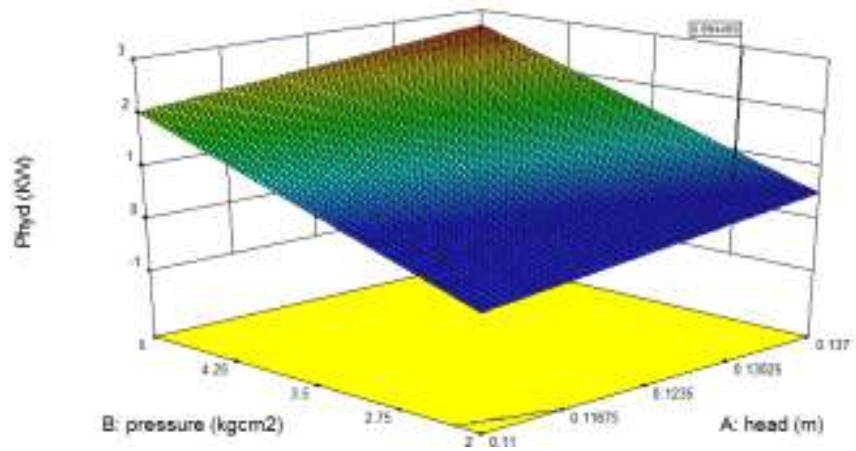

Fig16. Response curve for hydraulic power

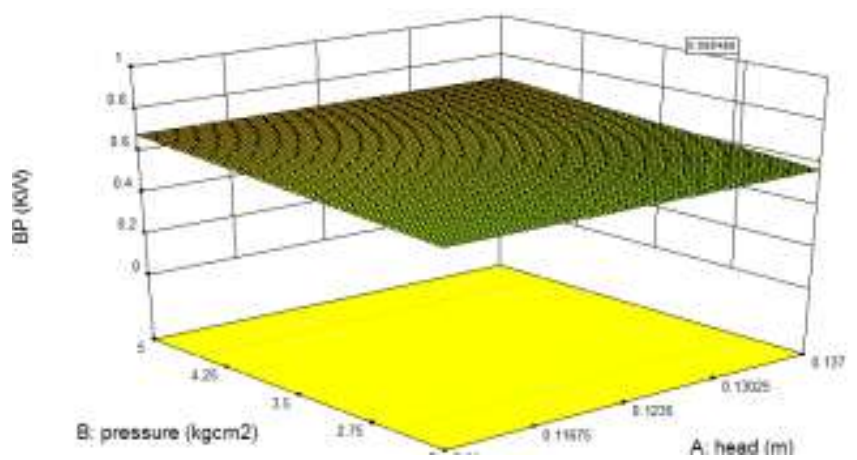

Fig17. Response curve for brake power

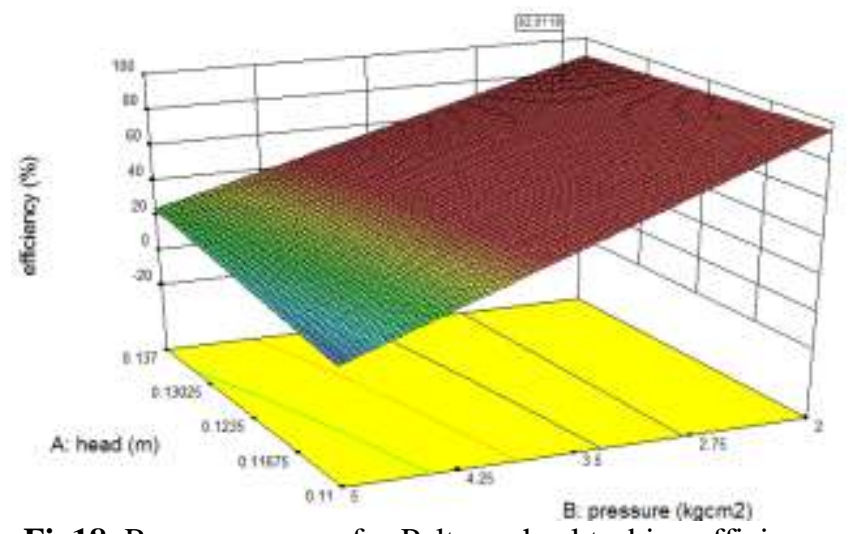

Fig18. Response curve for Pelton wheel turbine efficiency

The variation leads to the optimization of the factors which are shown in the graphs above. The optimal set developed is shown below: 


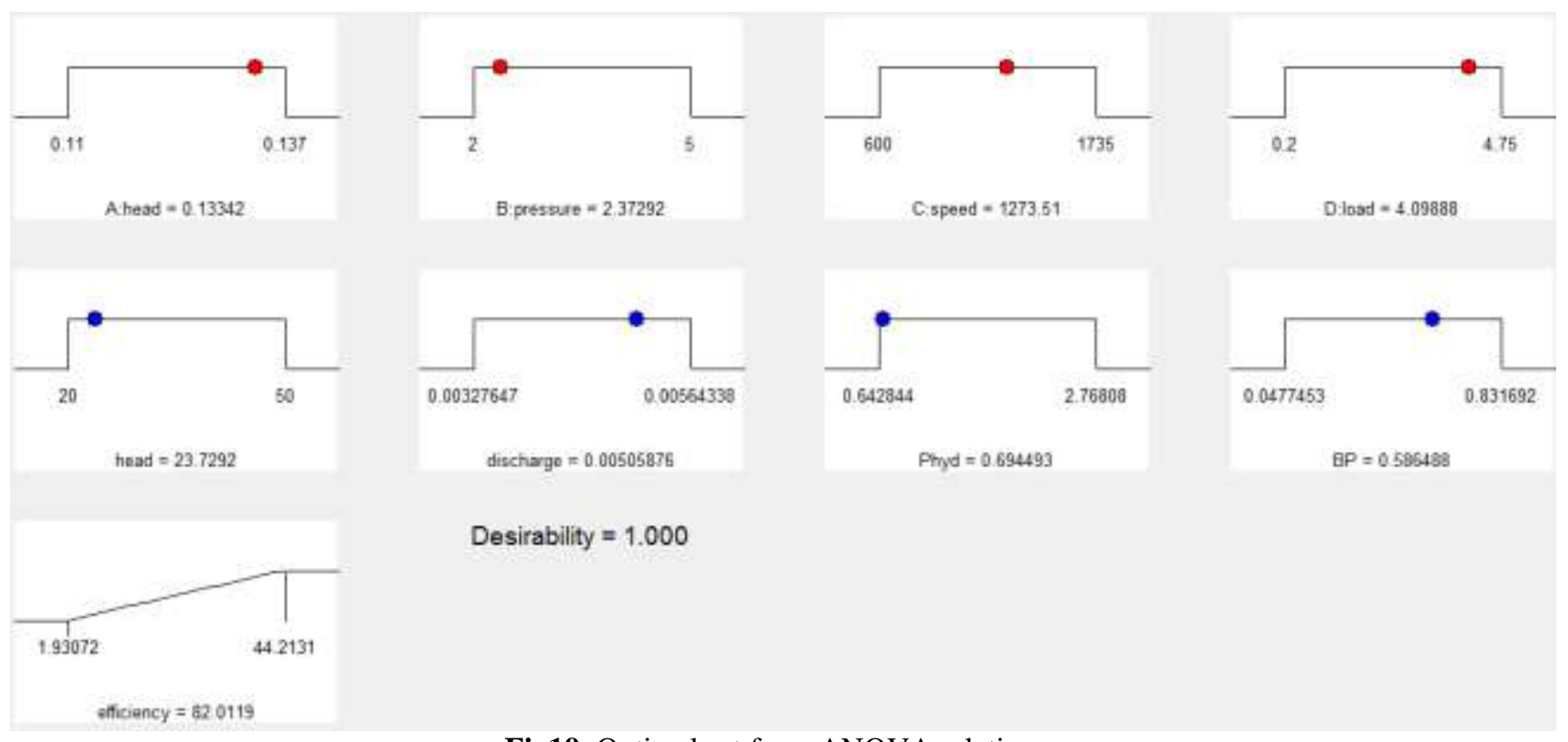

Fig19. Optimal set from ANOVA relations.

The optimal set obtained a desirability ratio of unity and a maximum efficiency of $82.01 \%$

\subsection{Pelton Wheel Test Rig Assessment}

The final part of the project is the assessment of the test rig from data obtained above. The set of inputs which deliver maximum efficiency from response surface method are fed into test rig and the performance is measuredaccordingly.

The efficiency gained from test rig is $65.33 \%$. Hence error between the set of readings is $16.68 \%$. This error can be accounted for due to friction and other losses.

\section{CONCLUSION}

Stochastic analysis was successfully carried out for Pelton wheel test rig. Response curves were drawn accordingly to identify optimal responses.

The comparison of response surface efficiency and Pelton test rig efficiency displayed a marginal error of $16.68 \%$ the reasons for which are likely to be vibrations and frictional losses in the system. Further research and investigation will be carried out to determine the errors which are to be reduced to a minimum. The assessment of the Pelton Wheel test rig was successful carried out.

\section{REFERENCES}

[1] KADAMBI V., MANOHAR PRASAD: An Introduction to Energy Conversion VolIII, New Age International Publishers, New Delhi, 1997.

https://www.wikipedia.org/

[2] P. BALACHANDRAN. Fundamentals of Compressible Flow, Ist Edition, Prentice Hall of India, New Delhi, 2007.

[3] Gelpke, Viktor; Van Cleve, Aaron Howell, 1868- joint authorHydraulic turbines, their design and installation

[4] Oliver Knill, Probability Theory and Stochastic processes 